Check for updates

Cite this: RSC Adv., 2017, 7, 26974

\title{
Efficient fabrication methodology of wide angle black silicon for energy harvesting applications $\uparrow$
}

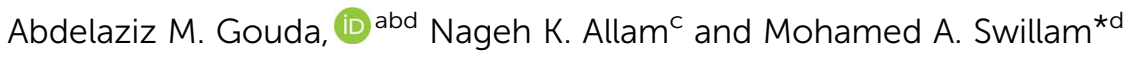

In this paper, we report an easy and relatively cost effective fabrication technique of a wide band omnidirectional antireflective black silicon surface based on silicon nanowires (SiNWs). An effective and economical one step silver electroless catalytic etching method in an aqueous solution of $\mathrm{AgNO}_{3}$ and $\mathrm{HF}$ is used for the synthesis of the black silicon surface. The formation mechanism for SiNW arrays is explained in terms of a localized nanoelectrochemical cell. The length and diameter of the nanowires were controllable as we found a commensurate relationship between dimensions and the etching time. Different sample sizes were used to prove the technique's large scale production potential. Wide range near zero reflection is reported in the visible region due to the strong trapping and antireflective properties in addition to a wide angle up to $\pm 60^{\circ}$. Raman scattering measurements confirmed the quantum size effect and phonon scattering in the fabricated structure with different diameters. A black silicon surface based on solid and porous SiNWs shows promising potential for photovoltaic, optoelectronic and energy storage applications.
\end{abstract}

Received 27th March 2017 Accepted 10th May 2017

DOI: $10.1039 / \mathrm{c} 7 \mathrm{ra03568c}$

rsc.li/rsc-advances reflection losses which limit their efficiency and their use. ${ }^{4-7}$ Possible solutions are using AR coatings to suppress reflection and high aspect ratio nanostructures to increase absorption by increasing the optical path of photon. ${ }^{2,8,9}$ However, most common AR coatings are limited in use due to their chemical, thermal and mechanical instability with the thin film and their validity only for certain wavelengths and certain incident angles. ${ }^{2,9}$ The limitation of the traditional AR coatings for certain wavelength and angle range such as using $\mathrm{SiN}_{x}$ layer as AR coating on silicon based solar cells reduced the reflection from $40 \%$ to $6 \%$ only with normal incidence, is very common problem. ${ }^{9}$ Therefore, using AR coatings for wide wavelengths and angles range is inefficient, requires high cost for multilayer use and for a mechanical tracking system to follow the solar radiation at different angles., ${ }^{2,9}$

In the last few years, black silicon (B-Si) paved the way not only for highly efficient Si solar cells but also for a wide range of applications ranging from energy applications like lithium ion batteries, ${ }^{10}$ hydrogen production through water splitting, ${ }^{11}$ sensing applications ${ }^{\mathbf{1 2 , 1 3}}$ and photonic devices. ${ }^{\mathbf{1 4 - 1 6}} \mathrm{B}-\mathrm{Si}$ is widely preferred for solar energy harvesting applications due to its unique morphology. This morphology changes the effective index at Si-air interface thereby reducing the reflection and leading to increased absorption and increased short circuit current density of solar cell. ${ }^{17}$ The first solar cell based on black silicon had efficiency $1.6 \%{ }^{18}$ The optical absorption and the minority carrier lifetime are very important parameters in determining the efficiency of solar cells. ${ }^{19}$ More research and developments are still undertaken to offer better control on the solar cell parameters towards high efficiency till reach $22.1 \%{ }^{20}$ Therefore, researchers are looking for methods to produce

\footnotetext{
${ }^{a}$ Department of Physics, American University in Cairo, New Cairo 11835, Egypt. E-mail: Abdelaziz@aucegypt.edu

${ }^{b}$ Department of Physics, Faculty of Education, Ain Shams University, Roxy, Cairo, Egypt

${ }^{c}$ Energy Materials Laboratory (EML), Department of Physics, American University in Cairo, New Cairo 11835, Egypt.E-mail:nageh.allam@aucegypt.edu

${ }^{d}$ Nanophotonics Research Laboratory (NRL), Department of Physics, American University in Cairo, New Cairo 11835,Egypt.E-mail: M.swillam@aucegypt.edu

$\dagger$ Electronic supplementary information (ESI) available. See DOI: $10.1039 / \mathrm{c} 7 \mathrm{ra03568c}$
} 
black silicon on purpose, especially at low cost and high repeatability. Black silicon surfaces have been fabricated by texturing the Si surface or by fabricating different nanostructures such as nanopores and vertical nanostructures on $\mathrm{Si}$ surface. ${ }^{\mathbf{1 0 , 1 9 , 2 1}}$ Many fabrication techniques are stated for B-Si fabrication including pulsed laser irradiation, ${ }^{18,22}$ reactive ion etching, ${ }^{23}$ and the preferred electroless wet metal assisted chemical etching (EMWCE).$^{24}$ Some bottom-up techniques are also used such as vapour liquid solid (VLS), ${ }^{25,26}$ and chemical vapour deposition (CVD). ${ }^{27}$ However, they are not industrially preferred owing to their high cost and complicated fabrication steps. $^{21}$

Porous and non-porous SiNWs have gained much attention as they are fabricated from Si. Their unique dimensions with high aspect ratio for the solid wires and high porous surface area for the porous wires offer inimitable optoelectronic and thermoelectric properties ${ }^{\mathbf{2 8 , 2 9}}$ beside the ultimate common use of solid wires for photovoltaic applications. Therefore, fabricating SiNWs at low cost, compatible with large scale fabrication with controlled dimensions is of prime importance for these applications. The EMWCE method is simple, low cost as it doesn't need any clean room facilities or any expensive complex equipment, less time consuming, and ambient temperature fabrication compared with VLs, and with no metal contamination. ${ }^{6}$ It is also a solution-based process, enabling fabricating vertical high density crystalline SiNWs with large area. ${ }^{24}$ Moreover, EMWCE produces SiNWs array with identical crystal structure to the parent wafer. EMWCE can be done through two definite processes; the first process is the single step EMWCE where $\mathrm{Si}$ is etched in an aqueous solution of $\mathrm{HF}$ and $\mathrm{AgNO}_{3}$ to form SiNWs. ${ }^{1,30,31}$ The second process involves double step etching where $\mathrm{Ag}$ particles are first deposited using an aqueous solution of $\mathrm{HF}$ and $\mathrm{AgNO}_{3}$ then etching the Si coated with $\mathrm{Ag}$ particles in an aqueous solution of $\mathrm{HF}$ and $\mathrm{H}_{2} \mathrm{O}_{2} \cdot{ }^{6,24,32}$ Two-step etching process is explored extensively in the literature while the preferred single step etching process has not been fully explored.

In this paper, we present a novel approach to fabricate B-Si surface based on N-type meso-porous and non-porous SiNWs with near zero reflection $(>0.5 \%)$.

We previously reported our initial results using similar structures without optimization. ${ }^{33}$ Here, after optimization, we found that the anti-reflection properties resulted in a surface reflecting a maximum of only $0.6 \%$ of light at $60^{\circ}$ incident angle while in ref. 33 the surface reflected $1.5 \%$ of the incident light. The optimizations allowed a wider wavelength range and wider angle anti-reflection. In addition, here we report the full details of our novel approach based on single step EMWCE to fabricate the B-Si surface. We explain the different properties of our structure using new measurements such as X-ray diffraction (XRD), Raman spectroscopy, nitrogen absorption measurement and transmission electron microscope (TEM) images. Our B-Si surface is based on large area porous and non-porous vertically aligned crystalline dense SiNWs array with controlled length and diameter and is fabricated economically and in an efficient manner. The variation in length and diameter is done through the variation of the etching time at ambient temperature. The optical properties of the fabricated SiNWs are investigated, proving that our structure is B-Si. The effect of changing diameter of solid wires on the optical properties is studied in the visible range. The effect of porosity and surface roughness on the phonon confinement is studied through Raman scattering measurement. B-Si based on the fabricated structure through single step EMWCE have very low reflection losses, therefore solid-SiNWs fabricated through this method have potential for wide range of PV and optoelectronics applications while the porous-SiNWs have potential in energy storage application due to their large surface area. The main purpose of this study is pushing the limits towards high efficient B-Si solar cell through enhancing the optical properties in simple cost effective method.

\section{Experimental methods}

The material used in this work is N-type C-Si (100) phosphorous lightly doped with a resistivity of 1-10 $\Omega \mathrm{cm}$, medially doped (resistivity less than $0.3 \Omega \mathrm{cm}$ ) and heavily doped with resistivity less than $0.09 \Omega \mathrm{cm}$. Samples were cut into pieces of fixed dimensions of $1.5 \times 1.5 \mathrm{~cm}^{2}$. Samples size was kept fixed through the process for different etching time to avoid any influence of the size on the fabrication mechanism. Si samples were cleaned sequentially in ultrasonic bath of acetone, ethanol each for 5 min then piranha solution $\left(\mathrm{H}_{2} \mathrm{SO}_{4}: \mathrm{H}_{2} \mathrm{O}_{2}\right.$ in a ratio of $4: 1)(\mathrm{v} / \mathrm{v})$ for $10 \mathrm{~min}$ at $80{ }^{\circ} \mathrm{C}$ to remove organic and inorganic contamination. After each acid treatment, samples were rinsed with deionized (DI) water. Then the cleaned samples were immersed in an aqueous solution of $\mathrm{AgNO}_{3}(5 \mathrm{mM})$ and $\mathrm{HF}$ (3 M) for different periods (from $30 \mathrm{~min}$ to $60 \mathrm{~min}$ with time step of $5 \mathrm{~min}$ ) at room temperature. The etched samples are steeped in $\mathrm{HNO}_{3}(30 \% \mathrm{wt})$ for $20 \mathrm{~s}$ at room temperature to remove silver nanoparticles. Lastly, samples are rinsed with DI water and dried with nitrogen.

The morphology characteristics and the dimensions of the fabricated SiNWs were observed by Zeiss Ultra60 field emission scanning electron microscope (FESEM) and high resolution transmission electron microscope (HRTEM) JEM-2100, JEOL, Akishima-shi, Japan. Reflectance measurements of fabricated SiNWs were done using spectrophotometer (JASCO Corp., V570 , Rev. 1.00) from 400-800 nm wavelength range for normal incidence measurement. Reflectance measurements with different incident angles were done using a Perkin-Elmer Lambda (UV/Vis NIR) spectrophotometer with universal reflectance unit. X-ray diffraction (XRD) diffraction pattern for different dimensions SiNWs was done by using $\mathrm{Cu} \mathrm{K} \alpha 1$ radiation source $(\lambda=1.5406 \AA)$ to investigate the crystal structure. For the TEM, high-resolution TEM (HRTEM) SiNWs were scratched off from the substrates and spread into methanol and ultrasonicated for $15 \mathrm{~min}$ and then salvaged with copper grids. The characterizations were performed under the voltage of $200 \mathrm{kV}$. Raman measurements were performed for the fabricated structures using Raman microscope (ProRaman-L Analyzer) with an excitation laser beam wavelength of $532 \mathrm{~nm}$. The pore size and the surface area of the different doping level SiNWs were measured using nitrogen adsorption isotherm. Porosity 
percentage of the solid SiNWs is measured using Quantachrome mercury poremaster.

\section{Results and discussion}

\subsection{Solid SiNWs and porous SiNWs array formation mechanism}

The mechanism of forming solid and porous vertical dense SiNWs array can be explained in terms of using Ag nanoparticles to assist the etching process. The etching process based on localized microscopic electrochemical model was proposed by Peng et al. ${ }^{32}$ where galvanic displacement of $\mathrm{Si}$ occurs due to the reduction reaction of $\mathrm{Ag}^{+}$on Si surface. The complete formation mechanism can be simplified in three main steps as shown in Fig. 1. The process of etching is continued until the solution is drained or the process is terminated. ${ }^{4}$

The complete redox reactions through the process are:

$$
\mathrm{Ag}^{+}+\mathrm{e}^{-} \rightarrow \operatorname{Ag}(\mathrm{s})
$$

(Reduction of silver ions at cathode)

$$
\mathrm{Si}(\mathrm{s})+2 \mathrm{H}_{2} \mathrm{O}+4 \mathrm{~h}^{+} \rightarrow \mathrm{SiO}_{2}+4 \mathrm{H}^{+}
$$

(Oxidation of silicon at anode)

$$
\begin{gathered}
\mathrm{SiO}_{2}+6 \mathrm{HF} \rightarrow \mathrm{H}_{2} \mathrm{SiF}_{6}+2 \mathrm{H}_{2} \mathrm{O} \\
\mathrm{Si}(\mathrm{s})+\mathrm{Ag}+6 \mathrm{HF} \rightarrow \mathrm{Ag}(\mathrm{s})+\mathrm{H}_{2} \mathrm{SiF}_{6}+4 \mathrm{H}^{+}
\end{gathered}
$$

(The complete redox reaction)

By using N-type Si, charge transfer from the more negative valance band energy of $\mathrm{Si}$ to the redox potential of $\mathrm{Ag}$ is

a)
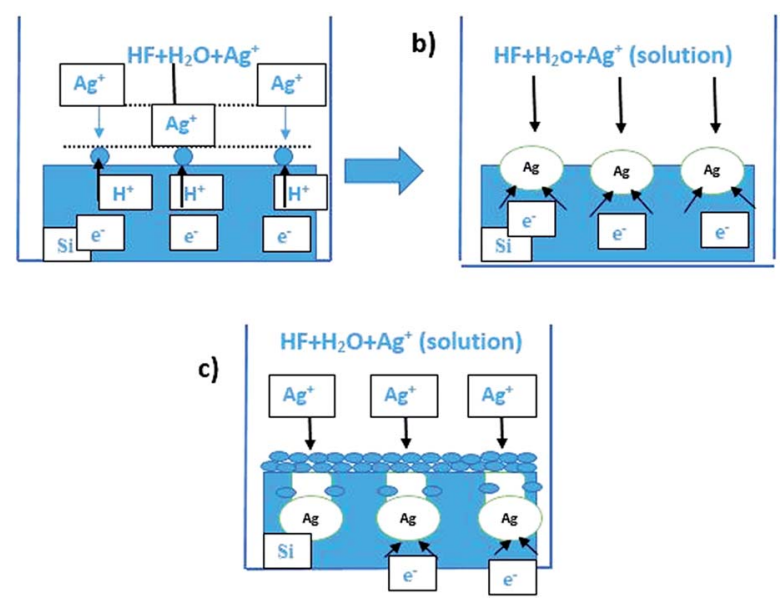

Fig. 1 Schematic representation of single step mechanism to form SiNWs; (a) $\mathrm{Ag}^{+}$ions are reduced to Ag nuclei through electron transfer from $\mathrm{Si}$ substrate to be oxidized into $\mathrm{SiO}_{x}$. (b) With increasing the etching time, Ag nuclei become larger and sink into Si substrate with the aid of HF which dissolute the oxide layer to facilitate the sinking of Ag particles. (c) The charges preferred to propagate vertically along $(\mathrm{Ag} / \mathrm{Si})$ interface which leads to speeding up the etching in the $(\mathrm{Ag} / \mathrm{Si})$ interface than the non $\mathrm{Ag}$ regions forming SiNWs. preferred. ${ }^{31} \mathrm{Ag}^{+}$ions are reduced into metallic $\mathrm{Ag}$ nanoparticles (AgNP) at the cathode while Si atoms at the surface are oxidized by the holes and form layer of $\mathrm{SiO}_{x}$. The $\mathrm{SiO}_{x}$ layer is then etched away by $\mathrm{HF}$ in form of $\mathrm{SiF}_{6}$, leaving pits on the surface where the Ag particles are trapped.

Remaining non-grown AgNP forms Ag Nano clusters (nanotrees) on the Si surface as shown in Fig. S1(a). $\uparrow$ The AgNPs sink downward due to the continuous galvanic displacement of $\mathrm{Si}$ and proceeds with time till forming straight vertical pores in the Si surface. AgNP are thermodynamically preferred to follow the surface defect states such as dangling bonds and dislocations which have less energy and facilitate their vertical growth. The etching direction of AgNP is determined by the crystal structure,,$^{\mathbf{4 , 2 4 , 3 4 , 3 5}}$ the plane orientation of the parent wafer, ${ }^{\mathbf{1 , 4 2 4}}$ the etching time, ${ }^{\mathbf{6}, 17}$ the etching temperature ${ }^{24,36}$ and the concentration of the etchant solution. ${ }^{30-32}$ The mechanism just described the formation of solid SiNWs using lightly and medially doped parent wafer. As dopant concentration increases, porosity and roughness increase due to several reasons as follows. The first reason is more thermodynamic driving force produced, increasing the rate of roughness and the rate of forming pores. ${ }^{31}$ The second reason is the higher level of doping lowering the energy barrier of charge transfer across Si surface which promotes electron tunnelling and the reaction rate. ${ }^{31}$ Therefore the rate of porosity and roughness increases during the process as some of the non-grown (not reduced) $\mathrm{Ag}^{+}$ions diffuse out of the SiNWs and settle on the side walls, forming internal pores which propagate laterally and induce side walls roughness. ${ }^{17,31,37}$

The previous kinetics steps are explained for $\mathrm{C}-\mathrm{Si}$ of plane orientation (100) which is preferred in all SiNWs etching synthesis methods. Although previous studies ${ }^{38,39}$ reported that (111) plane orientation of Si had lower Gibbs energy $=1240 \mathrm{~mJ}$ $\mathrm{m}^{-2}$ than (100) which had energy $=2130 \mathrm{~mJ} \mathrm{~m}^{-2}$, most of vertically aligned SiNWs were fabricated through (100) due to the following reasons:

(I) The covalent bonds of (100) plane are symmetrically less energy directed into the reactive solution which can be broken easily and form structures that favour etching Si atoms along this direction. ${ }^{40}$

(II) The density of hydrogen terminated $\mathrm{Si}$ atoms, which block the etching, is less in (100) Si compared to (111) Si. ${ }^{37,41}$

(III) The energy barrier of the plane orientation (100) is the smallest compared with the other planes which facilitate the charge transfer and the fabrication of vertical oriented wires in this direction. ${ }^{31}$

\subsection{The effect of the etching time on the morphology and dimensions of the fabricated structure}

Fig. 2(a), (b) and (j) show (45 tilt) FESEM images of the fabricated SiNWs array. To produce these solid vertical dense SiNWs array, we used the single step synthesis method with lightly doped Si at different etching times. The average length and diameter of the fabricated nanowires increases with increasing the etching time due to the increase of the etching rate as shown in TEM images; Fig. 2(e) and (f). Fig. 2(c) and (d) show the 


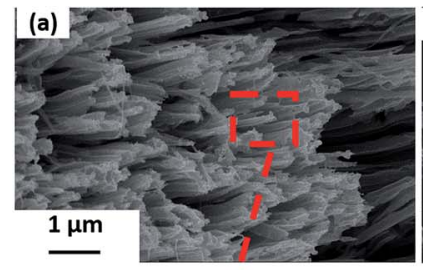

(e)
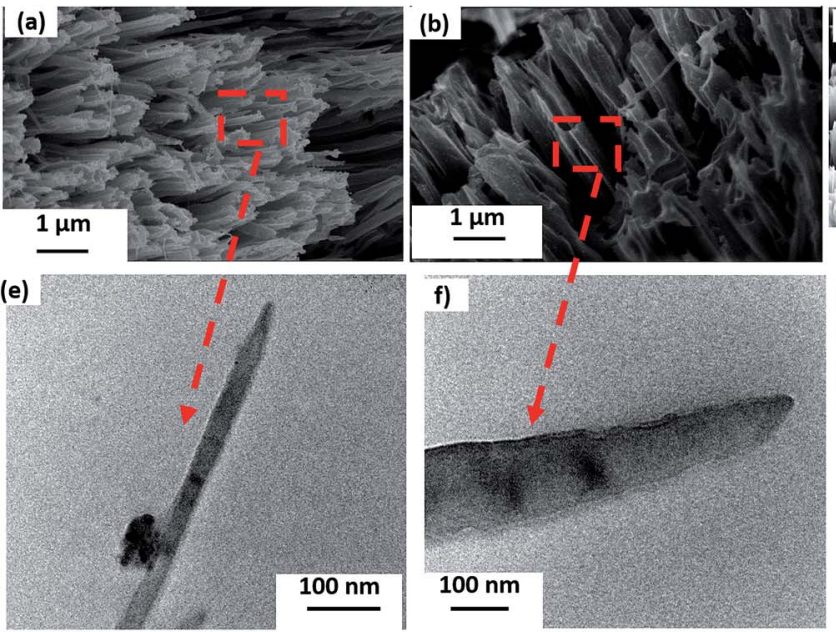
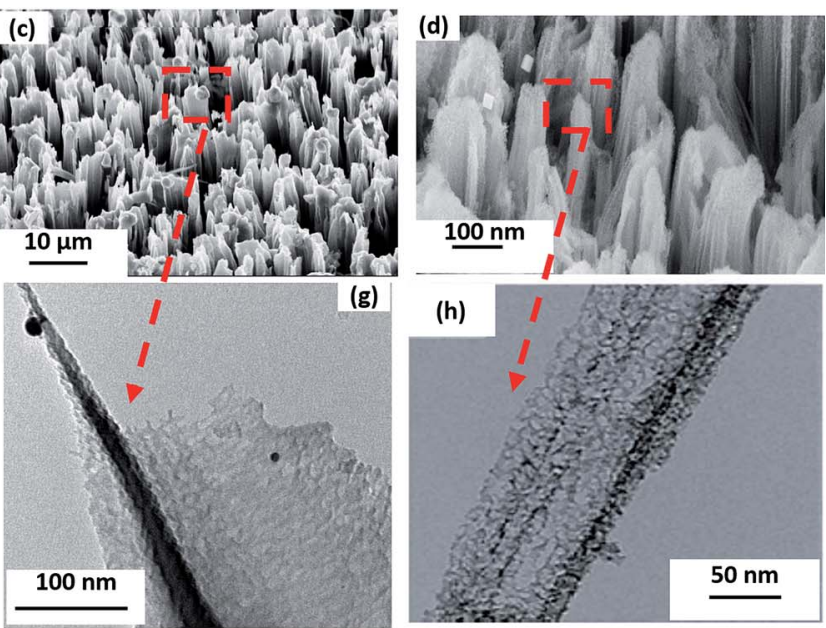

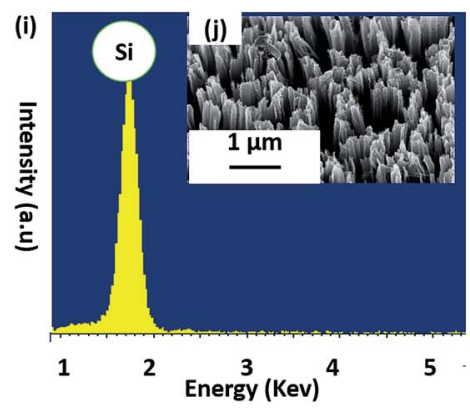

Fig. 2 FESEM images ( $a-d$ and $j)$ are ( $45^{\circ}$ tilt) vertical SiNWs array of different doping level at different etching time (a) lightly doped solid wires at $30 \mathrm{~min}$, (b) lightly doped solid wires at $60 \mathrm{~min}$, (c) medially doped porous wires at $30 \mathrm{~min}$, (d) heavily doped porous wires at $30 \mathrm{~min}$ and (i) EDX analysis with (j) FESEM image for lightly doped SiNWs at 50 min etching time after removal of Ag ions. (e)-(h) TEM images of SiNWs at different etching time (e) at lightly doped solid wires at $30 \mathrm{~min}$, (f) lightly doped solid wires at $60 \mathrm{~min}$ ( $\mathrm{g}$ ) medially doped porous wires at $30 \mathrm{~min}$ and (h) heavily doped porous wires at 30 min.

FESEM tilted morphology of the medially and heavily doped SiNWs; while medially doped SiNWs were vertical wires, heavily doped were benched straight wires with smaller diameter and more random porosity and roughness along the wire due to the high level of doping. The increase of the side wall roughness and the random pores are confirmed by TEM images as in Fig. 2(h). The wires start to aggregate together after $30 \mathrm{~min}$ etching time because the random pores reduce the mechanical strength of the wire and make them more fragile as in FESEM image Fig. 2(d) which could be related to the high level of doping.
In case of lightly doped vertical oriented SiNWs; the length is increased from 5 to $10.5 \mu \mathrm{m}$ corresponding to etching time increase from $30 \mathrm{~min}$ to $60 \mathrm{~min}$; the length is increased with a rate of $0.2 \mu \mathrm{m} \mathrm{min}^{-1}$ for the first $15 \mathrm{~min}$ then the rate is slowed down for the longer etching time to be $0.16 \mu \mathrm{m} \mathrm{min}^{-1}$ as shown in Fig. 3(b). The etching rate decreased for the long etching time ( $<45 \mathrm{~min}$ ) due to the decrease of density of $\mathrm{Ag}$ ions in solution after the $\mathrm{Ag}$ cluster is deposited over the sample and due to the decrease of HF concentration for long time etching. The average diameter is also increased from $250 \mathrm{~nm}$ to $1200 \mathrm{~nm}$ upon
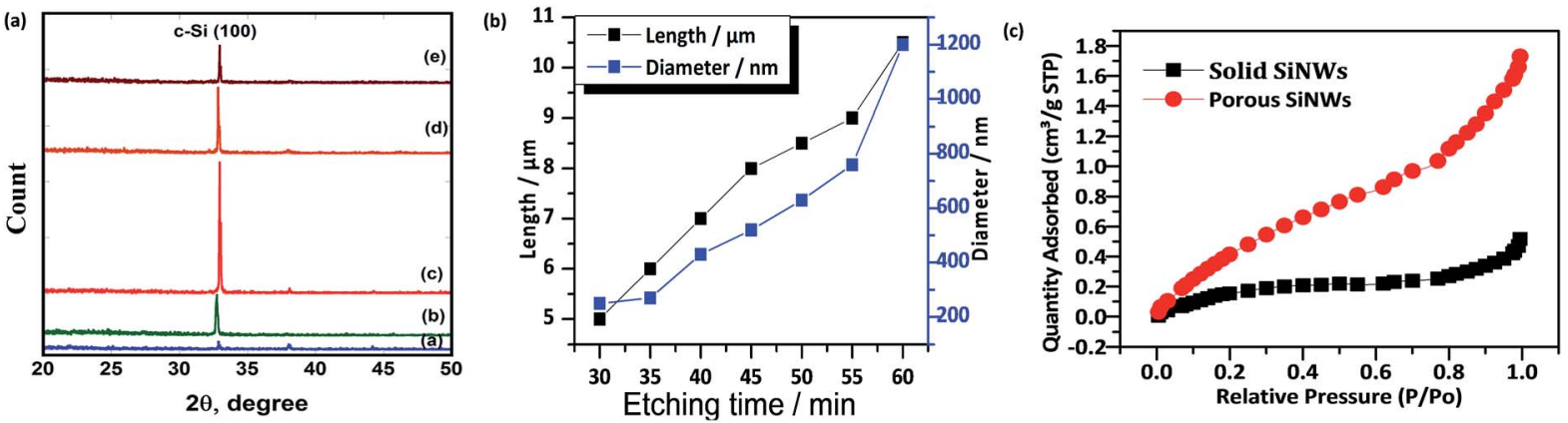

Fig. 3 (a) XRD patterns of the synthesized porous SiNWs (a) and non-porous SiNWs array at different etching time: (b) 30 min, (c) 40 min, (d) $50 \mathrm{~min},(\mathrm{e}) 60 \mathrm{~min}$, (b) the variation of the length and diameter of lightly doped SiNWs with etching time, (c) nitrogen adsorption measurement of porous and non-porous SiNWs. 
increasing etching time from $30 \mathrm{~min}$ to $60 \mathrm{~min}$ with an increasing rate of $31.6 \mathrm{~nm} \mathrm{~min}{ }^{-1}$.

This can be explained using one or more of the following:

(I) Aggregation of SiNWs which could be related to their flexibility. ${ }^{4}$

(II) Increasing van der Waals forces with increasing length and the etching time, which holds the wires together. ${ }^{4}$

(III) Surface tension force during the drying process. ${ }^{4}$

Fig. 3(a) shows that the fabricated structure has diffraction peaks observed around $33^{\circ}$ which could be related to the crystalline silicon (100) as the parent wafer. Therefore, we highlight that the single step fabrication method retains the crystal structure and the orientation of the parent wafer.

Nitrogen adsorption measurements are carried out for lightly and heavily doped SiNWs to confirm the porosity of samples and the pore diameter. Fig. 3(c) shows the nitrogen adsorption isotherm for solid lightly doped SiNWs and porous heavily doped SiNWs. The BET analysis yields a mean surface area of porous SiNWs $=2 \mathrm{~m}^{2} \mathrm{~g}^{-1}$, with total pore volume $=$ $0.002443 \mathrm{~cm}^{3} \mathrm{~g}^{-1}$ and a mean pore diameter $=5 \mathrm{~nm}$ compared to the solid wires of mean surface area $=0.71 \mathrm{~m}^{2} \mathrm{~g}^{-1}$ and total pore volume less than $0.000651 \mathrm{~cm}^{3} \mathrm{~g}^{-1}$ which confirms TEM images in Fig. 2(e), (f) and (h). The crystalline silicon is preferred in most of PV applications than amorphous silicon (a$\mathrm{Si}$ ) because a-Si has high degree of disorder and dangling bonds which make the carrier mobility very low with short life time. ${ }^{42}$

\subsection{The optical properties of the fabricated vertical SiNWs array at different etching time}

Fig. S2 $\uparrow$ shows different FESEM images of different samples dimensions starting from $0.8 \times 0.8 \mathrm{~cm}^{2}$ to $3 \times 3 \mathrm{~cm}^{2}$ are fabricated under the same fabrication conditions; etchant solution concentration and etching time (30 min). The vertical orientation and the surface coverage of the SiNWs does not change with changing the dimensions of the used samples which is a good proof that this method is valid also for large scale production. While increasing the surface area of the sample, the fabricated wires become shorter in length compared with the small surface area sample which leads to enhance the antireflective and trapping properties for large area samples. This could be related to the self-limiting as the rate of reaction depends on the concentration of the reactants; if the sample surface area is large, the concentration of the reactants and the rate of etching will decrease with time till HF consumes and the etching stops. ${ }^{4}$ The optical properties of the large area SiNWs array changes as in Fig. S3(b). $\dagger$ Reflection decreases with increasing surface area due to the shorter length making it a promising material for large scale PV devices. ${ }^{43}$

Fig. 4 shows the optical properties of different level of doping SiNWs; Fig. 4(a) compares between the reflectance spectra of the lightly doped, medially doped and heavily doped SiNWs with bare Si. The best antireflective properties comes from the porous heavily doped SiNWs as it was also reported that porosity could decrease the exciton diffusion length and enhance the charge separation due to the decrease of the silicon skeleton. ${ }^{\mathbf{4}}$ However, we found that for PV applications, porosity is undesirable Huang et $a l^{45}$ mentioned that the porosity of heavily doped SiNWs reduce the optical trapping through the solar irradiance spectrum (400-800 $\mathrm{nm}$ ) and weakens the mechanical strength of the wires. ${ }^{45}$ Therefore, porous SiNWs cannot be the best candidate for enhancing PV power conversion which could be explained due to the side wall roughness
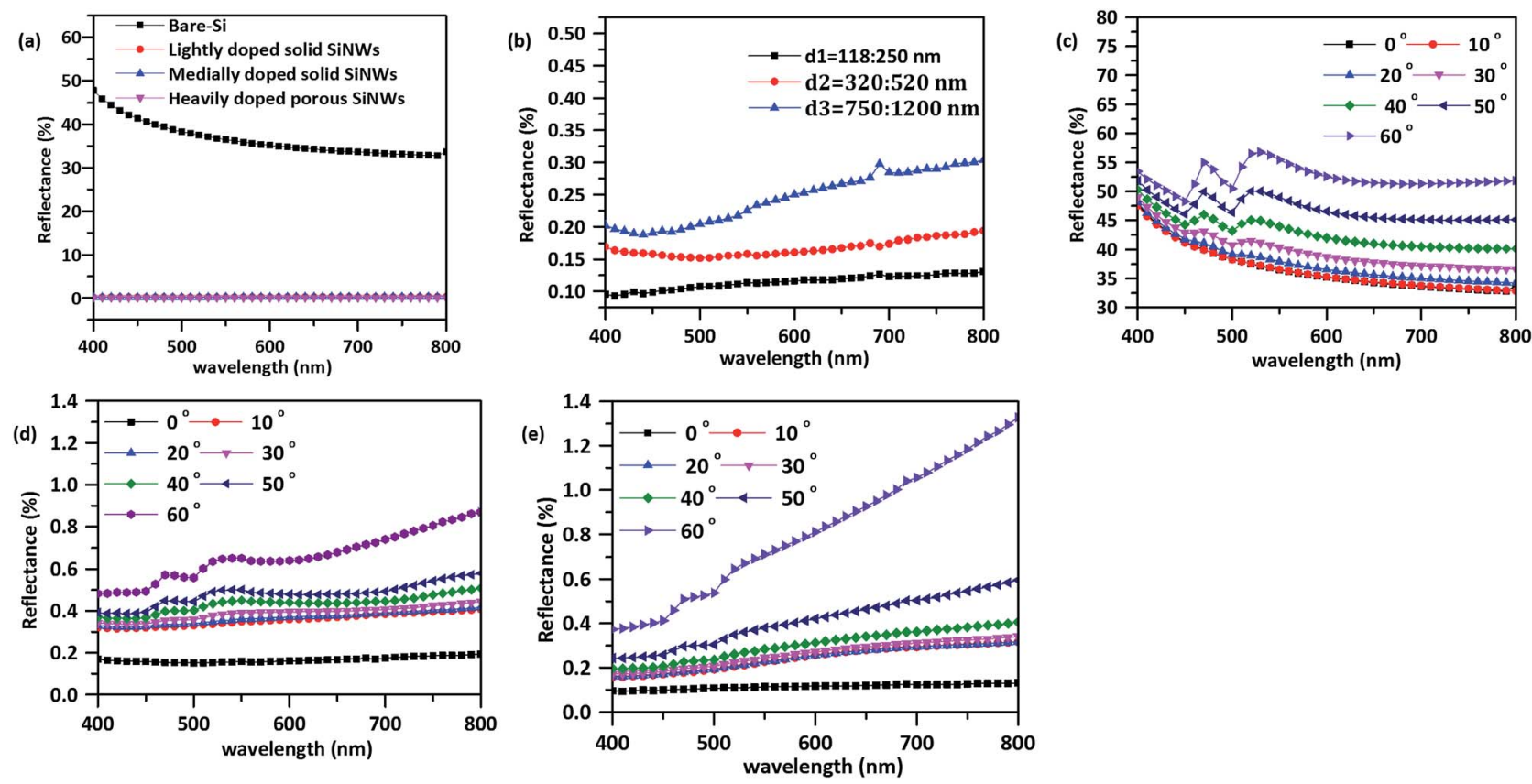

Fig. 4 (a) Reflection measurement of different level of doping SiNWs compared with bare-Si, (b) variation of reflection with different diameter of solid SiNWs, (c) change of reflection with incident angle for bare Si substrate, (d) variation of reflection with incident angle for solid SiNWs of diameter $=118-250 \mathrm{~nm}$ and (e) variation of reflection with incident angle for solid SiNWs of diameter $=320-520 \mathrm{~nm}$. 
and porosity of the porous wires. The gradual change in porosity and roughness along wires changes the effective index of the wires gradually with the incident wavelength to reflect the light at different paths. ${ }^{\mathbf{1 1 8}}$ The path difference between the incident and reflected wave in every case satisfies the destructive interference condition and destructs any incident light at Si-air interface, therefore the porous wires has very promising antireflective property with reduced trapping property. The reduced trapping property of the porous wires also could be attributed to the extra ordinary transmission. When the electric field of the incident light is perpendicular to the cross-line of the mesopores along wires which makes the optical peak is insensitive with the incident angles and sensitive with the angle between the electric field of the incident light to the cross-line of mesopores. ${ }^{46,47}$ The medially and lightly doped SiNWs show a very promising antireflective, absorption and trapping properties that enable them to enhance the efficiency of energy conversion applications. They are suitable for these applications because that are solid, vertically oriented wires without aggregation compared with the porous wires. The optical properties of the lightly doped SiNWs and the medially doped SiNWs for the same fabrication conditions are very consistent; the wires are of the same dimensions and with the same spacing under the same fabrication conditions. Due to the amenable promising optical properties of the solid lightly doped SiNWs, the fabrication method is examined for large scale production.

Solid SiNWs with different diameters are characterized to study the influence of changing the diameter and the length on the optical properties. Fig. 4(b) shows the effect of changing the diameter of the fabricated SiNWs array on the reflection. The vertical dense SiNWs array show a strong antireflection properties compared to the bare $\mathrm{Si}$ as the reflection of different diameters SiNWs is nearly zero reflectance. The strong antireflection properties of the vertical dense SiNWs resulted from tuning the diameter which changes the effective index of the interacting medium with light. ${ }^{48,49}$ Therefore, light is trapped along the structure which increases the optical path of photons due to multiple reflections occurring between arrays. ${ }^{50,51}$ Changing the diameter of the SiNWs array also changes the optical properties of the array. Due to the change of the light confinement which decreases with increasing the diameter. ${ }^{\mathbf{8} 48}$ Large diameter SiNWs array exhibits a perfect antireflective properties in the long wavelength region. This strong antireflective property through long wavelength due to the matching between the wavelength of the incident light and the dimensions of the SiNWs array enhances the light trapping and elongate the optical path of photons and decreases the reflection. ${ }^{\mathbf{8} 48,50,51}$ On the other hand, if the dimensions of the SiNWs array is greater than the wavelength of the light, most of the incident light is reflected which explained the bad reflection of the large diameter in the short wavelength. Fig. 4(c) and (e) shows the dependence of the reflection on the incident angle where the incident angle increases up to $\pm 60^{\circ}$ reflection increases due to vertical alignment of the dense SiNWs array as light becomes more oblique for large angles especially after $50^{\circ}$. The strong broad band omnidirectional antireflective properties and the angular independence comes from the variety of diameters with the gradual change of the effective index which enhances the light trapping and suppresses reflection. ${ }^{9}$

\subsection{Raman measurement for solid and porous SiNWs array}

Raman scattering measurements has been used as an efficient method for determining the optical and acoustic phonons modes in SiNWs array. ${ }^{6}$ It is also a good evidence for the crystal (a)

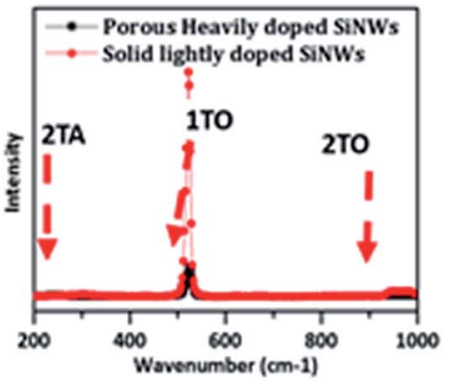

(d)

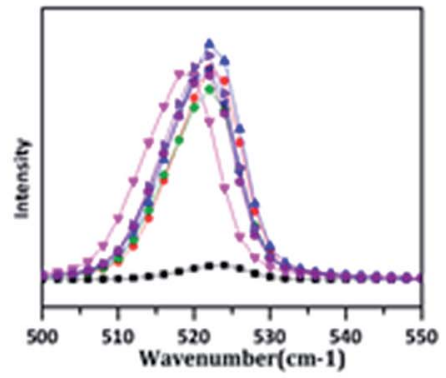

(b)
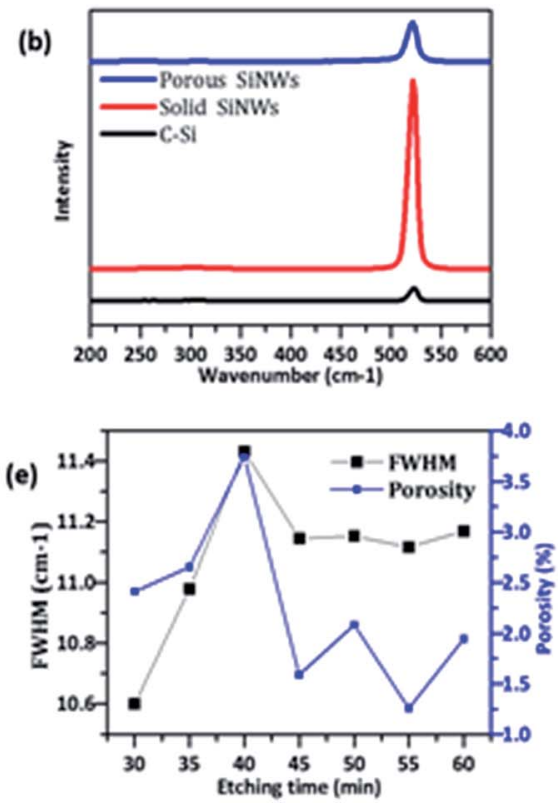

(c)

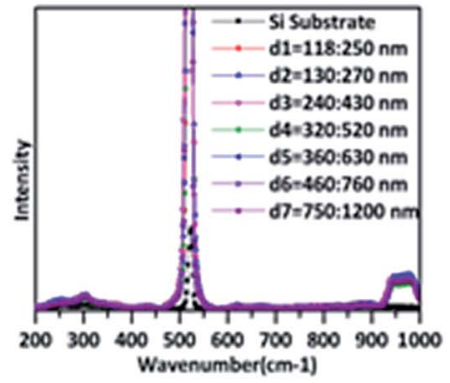

Fig. 5 (a) Raman spectra of the porous SiNWs and the solid SiNWs, (b) (1TO) Raman peak shift in porous and solid wires compared with C-Si, (c) Raman peaks of solid SiNWs with different diameter (d) 1TO peak shift in solid SiNWs of different diameter (e) the (FWHM) of the (1TO) and porosity of the different diameter solid SiNWs. 
size, crystallinity, and some scattering motivators like defects, porosity, and surface roughness. ${ }^{\mathbf{4 9 , 5 2 - 5 8}}$ In these measurements, the minimum laser power was used to reduce its heating effect on the measurements. ${ }^{58}$ Fig. 5(a) and (b) shows the Raman peak for C-Si at $524 \mathrm{~cm}^{-1}$ with full width half maximum (FWHM) of $10.26 \mathrm{~cm}^{-1}$ resulted from the first order optical phonon. On the other hand solid and porous SiNWs arrays have many Raman peaks; the main peak at $522 \mathrm{~cm}^{-1}$ corresponds to the first order transverse optical mode (1TO) is shifted down toward lower frequency than the C-Si. A broad peak at $296 \mathrm{~cm}^{-1}$ corresponds to the second order transverse acoustic mode (2TA) and the other broad peak at $940 \mathrm{~cm}^{-1}$ can be attributed to the second order transverse optical phonon mode (2TO). ${ }^{58}$ The possible reasons for the down shift in the first transverse optical phonon mode for different diameter of solid SiNWs as in Fig. 5(c). Noting that increasing the diameter, shifting the peak to higher wavenumber due to the following:

(1) Rise in temperature; in high doping SiNWs with diameter greater than Bohr radius have reduced the band gap and increasing the power absorbed which raises the temperature and shift the peak. ${ }^{57,58}$

(2) Higher carrier density. ${ }^{58}$

(3) The quantum confinement effect and stresses in the nanostructure which is our case. ${ }^{58}$

Controlling the internal structure of SiNWs array through the porosity by changing the etching rate can change the roughness of the SiNWs and allow quantum confinement tunability of SiNWs. ${ }^{56-58}$ Fig. 5(d) shows the change of the porosity of the fabricated SiNWs array with changing the etching time which clearly changes the roughness and the phonons confinement effect as FWHM takes the same trend as the porosity, the more porous SiNWs array contains, the greater shift for 1TO peak and more (FWHM) compared to less porous SiNWs array. ${ }^{58}$ The crystallinity of the SiNWs array decreases with increasing the diameter which results in shifting 1 TO peak to lower frequencies. When the crystallinity decreases, the momentum conservation will be relaxed $(q \neq 0)$ and Raman active modes are not limited to the centre of the Brillouin zone. ${ }^{52,53}$ Increasing the etching time change the diameter and the porosity of the SiNWs which decreases the crystalline size and enlarge frequency shift and broaden peaks. This result confirms the theoretical model proposed by Richter et al. and Campbell et al. ${ }^{54,55}$ The first order Raman spectrum can be described by the following equations:

$$
\Delta \omega=-A\left(\frac{a}{D}\right) \gamma
$$

$(\omega)$ is the frequency mode shift, $(D)$ is the SiNWs diameter, $(a)$ is the lattice constant of silicon ( $a=0.543 \mathrm{~nm})$, and $A=47.41$ $\mathrm{cm}^{-1}$ and $\gamma=1.44$ are fit factors which change with changing SiNWs diameter.

$$
I(\omega)=\int \frac{d^{3} q|c(0, q)|^{2}}{[\omega-\omega(q)]^{2}+\left(\frac{\Gamma_{0}}{2}\right)^{2}}
$$

where $(q)$ is the photon wave vector, $\omega(q)$ is the phonon dispersion curve and $(\Gamma)$ is the geometrical sum of the inverse life time of zone centre phonons and $c$ is the weighting function. The weighting function chose as by physical arguments. ${ }^{54,55}$ Moreover, the enhancement of the first order Raman spectrum of SiNWs array compared to C-Si may be attributed to these probable reasons:

(1) Decreasing the remaining area of silicon after etching which increase the transmitted excitation intensity into the material ignoring the losses due to diffuse scattering. ${ }^{57}$

(2) Enhancing the light excitation due to interaction between Raman back scatter and nano-interstice surface between SiNWs array which is known as the plasmonic effect of SiNWs.

The methods in ref. 8 and 20 are able to reach $22 \%$ independent of fabrication method. For our black silicon we can remove any defect layer using simple alkaline or acidic wet etching method in addition to passivating the surface to increase the life time of minority charge carriers. ${ }^{19}$ Therefore we can expect that a full solar cell based on B-Si reported here will have the same efficiency as ref. 8 and 20 if they have the same optical properties. We have better reflectivity less than $0.5 \%$, therefore we expect better efficiency.

The state of art of our work is the fabrication of porous and non-porous SiNWs in one step electroless, low cost and low energy required method and also tuning the dimensions of our nanostructures till we get very low reflectivity less than $0.5 \%$ better than the reported in previous studies ${ }^{\mathbf{1 8 , 2 0}}$ for most efficient solar cells based on B-Si. Our black silicon substrates are aiming to be used an efficient active material in solar cells fabrication as we have better wide band and angle antireflectivity without ARC and mechanical tracking system.

\section{Conclusions}

We have elucidated a cost effective large scale catalytic electroless solution based method for fabricating black silicon based on solid and porous vertical crystalline dense SiNWs. Large scale vertical aligned mesoporous crystalline SiNWs array are fabricated through single step silver assisted wet chemical etching in an effective and low cost and time method compared with the previous fabrication techniques. All XRD diffraction patterns confirms the crystallinity of the solid and porous SiNWs. The diameter and the length of solid wires increases with increasing the etching time while the porous wires benched after $30 \mathrm{~min}$ etching time. The nitrogen adsorption measurements confirm the porosity and the large surface area of porous wires compared with the solid wires. The fabrication method is valid for large scale production with promising optical properties which make the large scale solid SiNWs array through Ag single step fabrication method is very favourable for PV applications. Both solid and porous wires show a strong wideband and angles antireflection properties but the solid wires trap the light more than the porous. The antireflective properties of solid wires are investigated for different diameters and it shows a strong dependence on the average diameters. The solid wires show a strong wide band omnidirectional antireflection properties up to $\pm 60^{\circ}$. The Raman spectra for solid and porous wires show the down shift in frequency compared with C-Si for different diameter SiNWs due to the 
porosity in the SiNWs array. The superior antireflection properties of our B-Si substrates are aiming to enhance the overall efficiency of B-Si solar cells. Solid and porous SiNWs may open the doors for a wide range of applications in PV, optoelectronics and energy storage applications.

\section{Acknowledgements}

This work was supported and funded by The American University in Cairo (AUC).

\section{Notes and references}

1 F. Bai, M. Li, R. Huang, Y. Li, M. Trevor and K. P. Musselman, RSC Adv., 2014, 4, 1794-1798.

2 J. Valenta and S. Mirabella, Nanotechnology and Photovoltaic Devices: Light Energy Harvesting with Group IV Nanostructures, CRC Press, 2015.

3 Y.-T. Lu and A. R. Barron, Phys. Chem. Chem. Phys., 2013, 15, 9862-9870.

4 S. K. Srivastava, D. Kumar, S. Schmitt, K. Sood, S. Christiansen and P. Singh, Nanotechnology, 2014, 25, 175601.

5 N. Han, F. Wang and J. C. Ho, Nanomater. Energy, 2012, 1, 417.

6 S. A. Razek, M. A. Swillam and N. K. Allam, J. Appl. Phys., 2014, 115, 194305.

7 L. J. Chen, J. Mater. Chem., 2007, 17, 4639-4643.

8 J. Oh, H.-C. Yuan and H. M. Branz, Nat. Nanotechnol., 2012, 7, 743-748.

9 J. Cai and L. Qi, Mater. Horiz., 2015, 2, 37-53.

10 C. K. Chan, H. Peng, G. Liu, K. McIlwrath, X. F. Zhang, R. A. Huggins and Y. Cui, Nat. Nanotechnol., 2008, 3, 31-35.

11 J. Oh, T. G. Deutsch, H.-C. Yuan and H. M. Branz, Energy Environ. Sci., 2011, 4, 1690-1694.

12 R. Gamal, Y. Ismail and M. A. Swillam, J. Opt., 2015, 17, 045802.

13 M. Miu, I. Kleps, L. Pavesi, F. Craciunoiu, T. Ignat, A. Dinescu and M. Simion, Nanostructured silicon for optical biosensors. In Semiconductor Conference, IEEE, 2007, vol. 2, pp. 345-348.

14 F. Qian, S. Gradecak, Y. Li, C.-Y. Wen and C. M. Lieber, Nano Lett., 2005, 5, 2287-2291.

15 M. Thönissen, M. G. Berger, M. Krüger, S. Billat, R. ArensFischer, O. Glück, H. Lüth, S. Hilbrich, W. Theiss and P. Grosse, Optical interference filters made of porous silicon, MRS Online Proceedings Library Archive, 1996, vol. 431.

16 M. Steglich, M. Zilk, A. Bingel, C. Patzig, T. Käsebier, F. Schrempel, E.-B. Kley and A. Tünnermann, J. Appl. Phys., 2013, 114, 183102.

17 D. Kumar, S. K. Srivastava, P. K. Singh, K. N. Sood, V. N. Singh, N. Dilawar and M. Husain, J. Nanopart. Res., 2010, 12(6), 2267-2276.

18 T. Sarnet, J. E. Carey, and E. Mazur, From black silicon to photovoltaic cells, using short pulse lasers, in AIP
Conference Proceedings, ed. C. Phipps, 2012, vol. 1464, pp. 219-228.

19 M. Otto, M. Algasinger, H. Branz, B. Gesemann, T. Gimpel, K. Füchsel, T. Käsebier, S. Kontermann, S. Koynov and X. Li, Adv. Opt. Mater., 2015, 3, 147-164.

20 H. Savin, P. Repo, G. Von Gastrow, P. Ortega, E. Calle, M. Garín and R. Alcubilla, Nat. Nanotechnol., 2015, 10, 624-628.

21 X. Liu, P. R. Coxon, M. Peters, B. Hoex, J. M. Cole and D. J. Fray, Energy Environ. Sci., 2014, 7, 3223-3263.

22 Y.-H. Yang, S.-J. Wu, H.-S. Chiu, P.-I. Lin and Y.-T. Chen, J. Phys. Chem. B, 2004, 108, 846-852.

23 Y. Fu, A. Colli, A. Fasoli, J. Luo, A. Flewitt, A. Ferrari and W. Milne, J. Vac. Sci. Technol., B: Microelectron. Nanometer Struct.-Process., Meas., Phenom., 2009, 27, 1520-1526.

24 Z. Huang, N. Geyer, P. Werner, J. De Boor and U. Gösele, Adv. Mater., 2011, 23, 285-308.

25 R. Wagner and W. Ellis, Appl. Phys. Lett., 1964, 4, 89-90.

26 L. Latu-Romain, C. Mouchet, C. Cayron, E. Rouviere and J.-P. Simonato, J. Nanopart. Res., 2008, 10, 1287-1291.

27 S. R. Marthi, S. Sekhri and N. Ravindra, JOM, 2015, 67, 21542159.

28 B. Tian, X. Zheng, T. J. Kempa, Y. Fang, N. Yu, G. Yu, J. Huang and C. M. Lieber, Nature, 2007, 449, 885-889.

29 A. I. Hochbaum, R. Chen, R. D. Delgado, W. Liang, E. C. Garnett, M. Najarian, A. Majumdar and P. Yang, Nature, 2008, 451, 163-167.

30 A. G. Nassiopoulou, V. Gianneta and C. Katsogridakis, Nanoscale Res. Lett., 2011, 6, 1.

31 W.-K. To, C.-H. Tsang, H.-H. Li and Z. Huang, Nano Lett., 2011, 11, 5252-5258.

32 K. Peng, J. Hu, Y. Yan, Y. Wu, H. Fang, Y. Xu, S. Lee and J. Zhu, Adv. Funct. Mater., 2006, 16, 387-394.

33 A. M. Gouda, N. K. Allam and M. A. Swillam, Facile omnidirectional black silicon based on porous and nonporous silicon nanowires for energy applications, in Photonics North (PN), IEEE, 2016, p. 1.

34 C. Q. Lai, H. Cheng, W. Choi and C. V. Thompson, J. Phys. Chem. C, 2013, 117, 20802-20809.

35 Z. R. Smith, R. L. Smith and S. D. Collins, Electrochim. Acta, 2013, 92, 139-147.

36 D. Kumar, S. K. Srivastava, P. Singh, K. Sood, V. Singh, N. Dilawar and M. Husain, J. Nanopart. Res., 2010, 12, 2267-2276.

37 A. Najar, A. Slimane, M. N. Hedhili, D. Anjum, R. Sougrat, T. Ng and B. Ooi, J. Appl. Phys., 2012, 112, 033502.

38 R. Jaccodine, J. Electrochem. Soc., 1963, 110, 524-527.

39 J. J. Gilman, J. Appl. Phys., 1960, 31, 2208-2218.

40 M. Christophersen, J. Carstensen, S. Rönnebeck, C. Jäger, W. Jäger and H. Föll, J. Electrochem. Soc., 2001, 148, E267E275.

41 Z. Huang, T. Shimizu, S. Senz, Z. Zhang, X. Zhang, W. Lee, N. Geyer and U. Gösele, Nano Lett., 2009, 9, 2519-2525.

42 J. M. Pearce, N. Podraza, R. W. Collins, M. M. Al-Jassim, K. M. Jones, J. Deng and C. R. Wronski, J. Appl. Phys., 2007, 101(11), 114301. 
43 S. Thiyagu, H.-J. Syu, C.-C. Hsueh, C.-T. Liu, T.-C. Lin and C.-F. Lin, $R S C$ Adv., 2015, 5, 13224-13233.

44 A. I. Hochbaum, D. Gargas, Y. J. Hwang and P. Yang, Nano Lett., 2009, 9, 3550-3554.

45 W.-K. To, J. Fu, X. Yang, V. Roy and Z. Huang, Nanoscale, 2012, 4, 5835-5839.

46 T. W. Ebbesen, H. J. Lezec, H. Ghaemi, T. Thio and P. Wolff, Nature, 1998, 391, 667-669.

47 J. V. Coe, J. M. Heer, S. Teeters-Kennedy, H. Tian and K. R. Rodriguez, Annu. Rev. Phys. Chem., 2008, 59, 179-202.

48 K. Inoue, K. Ohtaka and S. Noda, in Photonic Crystals, Springer, 2004, pp. 211-236.

49 K. Adu, Q. Xiong, H. Gutierrez, G. Chen and P. Eklund, Appl. Phys. A: Mater. Sci. Process., 2006, 85, 287-297.

50 S. Jeong, E. C. Garnett, S. Wang, Z. Yu, S. Fan, M. L. Brongersma, M. D. McGehee and Y. Cui, Nano Lett., 2012, 12, 2971-2976.
51 K.-T. Park, Z. Guo, H.-D. Um, J.-Y. Jung, J. M. Yang, S. K. Lim, Y. S. Kim and J.-H. Lee, Opt. Express, 2011, 19, A41-A50.

52 J. Lim, K. Hippalgaonkar, S. C. Andrews, A. Majumdar and P. Yang, Nano Lett., 2012, 12, 2475-2482.

53 I. Ponomareva, D. Srivastava and M. Menon, Nano Lett., 2007, 7, 1155-1159.

54 H. Richter, Z. Wang and L. Ley, Solid State Commun., 1981, 39, 625-629.

55 I. Campbell and P. M. Fauchet, Solid State Commun., 1986, 58, 739-741.

56 B. Li, D. Yu and S.-L. Zhang, Phys. Rev. B: Condens. Matter Mater. Phys., 1999, 59, 1645.

57 C. Li, G. Fang, S. Sheng, Z. Chen, J. Wang, S. Ma and X. Zhao, Phys. E, 2005, 30, 169-173.

58 W. McSweeney, C. Glynn, H. Geaney, G. Collins, J. D. Holmes and C. O'Dwyer, Semicond. Sci. Technol., 2015, 31, 014003. 